\title{
Perfil de desempenho de técnicas parasitológicas na detecção de helmintíases em área endêmica do nordeste do Brasil
}

\author{
Performance profile of parasitological techniques in the detection of helminthiasis in an endemic \\ area of northeastern Brazil \\ Perfil de desempeño de técnicas parasitológicas en la detección de helmintiasis en una zona \\ endémica del noreste de Brasil
}

Alexrangel Henrique Cruz Santos ORCID: https://orcid.org/0000-0002-3667-5275 Universidade Federal de Sergipe, Brasil E-mail: alex_rangel2020@hotmail.com Sara Antônia Silva da Vitória ORCID: https://orcid.org/0000-0002-6844-050X Universidade Federal de Sergipe, Brasil E-mail: ant.sara0023@hotmail.com

Thayane Santos Siqueira ORCID: https://orcid.org/0000-0001-8370-0544 Universidade Federal de Sergipe, Brasil E-mail: thayane_siqueira@live.com

Ariel Oliveira Celestino

ORCID: https://orcid.org/0000-0003-3907-187X Universidade Federal de Sergipe, Brasil E-mail: biomedica.ariel@gmail.com

Amanda Francielle Santos ORCID: https://orcid.org/0000-0001-7694-4604 Universidade Federal de Sergipe, Brasil

E-mail: francyelly_amanda@hotmail.com Magno Pereira Prado ORCID: https://orcid.org/0000-0003-1848-7219 Universidade Federal de Sergipe, Brasil Email: magnoprado@yahoo.com.br

Mariana do Rosário Souza

ORCID: https://orcid.org/0000-0002-3139-8483 Universidade Federal de Sergipe, Brasil E-mail: mariana_do_rs@hotmail.com

Beatriz Almeida Santos

ORCID: https://orcid.org/0000-0003-1527-615X Universidade Federal de Sergipe, Brasil

E-mail: beatriz.enf12@gmail.com

Luiz André Santos Silva

ORCID: https://orcid.org/0000-0003-1607-8649 Universidade Federal de Sergipe, Brasil E-mail: luizandressilva@yahoo.com

Ítalo Fernando Lisboa de Melo

ORCID: https://orcid.org/0000-0002-1754-8561 Universidade Federal de Sergipe, Brasil

E-mail: italo-lisboa@hotmail.com

Vinícius Barbosa dos Santos Sales ORCID: https://orcid.org/0000-0002-0697-395X Universidade Federal de Sergipe, Brasi

E-mail:vbarbosa77@gmail.com

Luana Santos Costa

ORCID: https://orcid.org/0000-0001-9972-6415 Universidade Federal de Sergipe, Brasil

E-mail: luanacosta159@gmail.com

Gleidson Felipe Hilario de Jesus

ORCID: https://orcid.org/0000-0003-4902-4321 Universidade Federal de Sergipe, Brasil E-mail: gleidson.hilario@hotmail.com 


\author{
José Rodrigo Santos Silva \\ ORCID: https://orcid.org/0000-0002-1918-7122 \\ Universidade Federal de Sergipe, Brasil \\ E-mail: rodrigo.ufs@gmail.com \\ Luciene Barbosa \\ ORCID: https://orcid.org/0000-0003-2026-994X \\ Universidade Federal de Sergipe, Brasil \\ E-mail: lucienebarb@bol.com.br
}

\begin{abstract}
Resumo
O estudo visa comparar a eficácia e a sensibilidade de três técnicas parasitológicas no diagnóstico das helmintíases em uma área endêmica no nordeste do Brasil. Trata-se de um estudo epidemiológico, descritivo e comparativo, o mesmo foi realizado no município de Maruim, que fica aproximadamente a $30 \mathrm{~km}$ de da capital do estado de Sergipe, a cidade de Aracaju. Foram distribuídos kits e coletadas 3 amostras diárias entre os dias 17 a 20 de setembro de 2018, posteriormente as amostras foram transportadas até o laboratório de Entomologia e Parasitologia Tropical da Universidade Federal de Sergipe para realizar análise macroscópica, aplicando o método de Kato-Katz, Hoffman, Pons e Janer, e o método de Ritchie. Foi encontrado uma maior prevalência de casos positivos para Schistosoma mansoni (48,12\%), ancilostomídeos (4,38\%) e Hymenolepis nana $(2,50 \%)$ quando analisado sob a técnica de Kato Katz, os helmintos Enterobius vermicularis (5,62\%), Trichuris trichiura (36,25\%) e Strongyloides stercoralis $(5,00 \%)$ mostrou-se mais encontrado quando utilizado a técnica de Ritchie. As técnicas se mostraram bastante eficientes na identificação dos parasitas, porém nem todos os parasitos foram identificados simultaneamente pelas técnicas. Com base nesta informação, conclui-se que uma técnica pode complementar a outra no diagnóstico parasitário aumentando assim sua sensibilidade.
\end{abstract}

Palavras-chave: Doenças tropicais negligenciadas; Saúde pública; Determinantes Sociais da Saúde.

\begin{abstract}
The study aims to compare the effectiveness and sensitivity of three parasitological techniques in the diagnosis of helminthiasis in an endemic area in northeastern Brazil. This is an epidemiological, descriptive and comparative study, carried out in the municipality of Maruim, which is approximately $30 \mathrm{~km}$ from the capital of the state of Sergipe, the city of Aracaju. Kits were distributed and 3 samples were collected daily between September 17th and 20th, 2018, later the samples were transported to the Entomology and Tropical Parasitology laboratory of the Federal University of Sergipe to perform macroscopic analysis, applying the Kato-Katz method, Hoffman, Pons and Janer, and the Ritchie method. A higher prevalence of positive cases for Schistosoma mansoni (48.12\%), hookworms $(4.38 \%)$ and Hymenolepis nana $(2.50 \%)$ was found when analyzed using the Kato-Katz technique, the helminths Enterobius vermicularis (5 .62\%), Trichuris trichiura (36.25\%) and Strongyloides stercoralis (5.0\%) were found to be more frequent when using the Ritchie technique. The techniques proved to be very efficient in identifying the parasites, but not all parasites were identified simultaneously by the techniques. Based on this information, it is concluded that one technique can complement the other in the parasitic diagnosis, thus increasing its sensitivity.
\end{abstract}

Keywords: Neglected tropical diseases; Public health; Social Determinants of Health.

\title{
Resumen
}

El estudio tiene como objetivo comparar la efectividad y sensibilidad de tres técnicas parasitológicas en el diagnóstico de helmintiasis en una zona endémica del noreste de Brasil. Se trata de un estudio epidemiológico, descriptivo y comparativo, realizado en el municipio de Maruim, que se encuentra aproximadamente a $30 \mathrm{~km}$ de la capital del estado de Sergipe, la ciudad de Aracaju. Se distribuyeron kits y se recolectaron 3 muestras diariamente entre el 17 y 20 de septiembre de 2018, posteriormente las muestras fueron transportadas al laboratorio de Entomología y Parasitología Tropical de la Universidad Federal de Sergipe para realizar análisis macroscópicos, aplicando el método Kato-Katz, Hoffman, Pons y Janer, y el método Ritchie. Se encontró una mayor prevalencia de casos positivos para Schistosoma mansoni $(48,12 \%)$, anquilostomas $(4,38 \%)$ e Hymenolepis nana $(2,50 \%)$ cuando se analizaron mediante la técnica de Kato-Katz, los helmintos Enterobius vermicularis (5,62\%), Trichuris trichiura (36,25\%) y Strongyloides stercoralis $(5,0 \%)$ resultaron ser más frecuentes cuando se utilizó la técnica de Ritchie. Las técnicas demostraron ser muy eficientes para identificar los parásitos, pero no todos los parásitos fueron identificados simultáneamente por las técnicas. Con base en esta información se concluye que una técnica puede complementar a la otra en el diagnóstico parasitario, aumentando así su sensibilidad.

Palabras clave: Enfermedades tropicales desatendidas; Salud pública; Determinantes Sociales de la Salud.

\section{Introdução}

As helmintíases classificam-se em dois grandes grupos também chamado de filos, os Nemathelminthes (vermes com corpo cilíndricos) e Platyhelminthes (vermes com corpo achatado dorsoventral). Alguns desses parasitam humanos, 
normalmente acometem o trato gastrointestinal principalmente em crianças. Essas helmintíases compõem o grupo das doenças tropicais negligenciadas, uma vez que, são mais frequentes em países subdesenvolvidos ou em desenvolvimento, com clima tropical e ainda, estão ligadas baixas condições socioeconômicas, ambientais e sanitárias, bem como fatores socioculturais de umas determinadas regiões do brasil e no mundo (Amarante et al., 2014; Silva et al., 2020; Gil et al., 2013; Mota et al., 2020)

Normalmente a transmissão desses parasitos ocorre principalmente, pela via oral-fecal, mas também pode ocorrer pela via percutânea devido a hábitos como andar descalço e por formas recreativas em coleções hídricas que acabam proporcionando contato com as formas infectante do parasito (Santos et al., 2020).

A identificação dos parasitos em fezes é feita rotineiramente por variadas técnicas de diagnóstico, que deve ter alta sensibilidade (diagnóstico correto e preditivo positivo), uma vez que o tratamento específico fica dependente dessas condições (SILVA et al., 2020). O exame consiste em um procedimento para a investigação das funções digestivas, seja pela metodologia macroscópica ou microscópica, possibilitando determinar possíveis síndromes (Lima et al., 2020).

Mesmo diante de inúmeros métodos quantitativos e qualitativos de diagnóstico, muitos ainda recebem críticas por suas limitações, complexidade de técnica e baixa sensibilidade, restringindo sua utilização na rotina de alguns laboratórios (SILVA et al., 2020). O diagnóstico clínico é impreciso, pois baseia-se em manifestações clínicas, que, nesse caso, podem variar desde quadros assintomáticos a outros de sintomatologias inespecíficas, tais como diarreia, náuseas, desconforto abdominal, dentre outros. Portanto, o diagnóstico laboratorial desempenha um papel importante no diagnóstico das infecções/doenças parasitárias, sendo a chave para a seleção da conduta terapêutica (Azevedo et al., 2017).

Alguns autores corroboram essas ideias e abordaram que nenhum teste é considerado $100 \%$ sensível para o diagnóstico. A sensibilidade no diagnóstico aumenta de acordo com a quantidade de amostras fecais analisadas podendo chegar a $100 \%$ com o uso de sete amostras, o que muitas vezes se torna um processo diagnóstico demorado e oneroso (Silva et al., 2020; Mota et al., 2020). Com base neste pressuposto, o presente estudo visa comparar a eficácia e a sensibilidade de três técnicas parasitológicas no diagnóstico das helmintíases em uma área endêmica no nordeste do Brasil.

\section{Metodologia}

Trata-se de um estudo epidemiológico, descritivo e comparativo, com o intuito de verificar a eficácia e a sensibilidade de três técnicas parasitológicas no diagnóstico das helmintíases. O mesmo foi realizado no município de Maruim, que fica aproximadamente a $30 \mathrm{~km}$ de da capital do estado de Sergipe, a cidade de Aracaju.

O município de maruim se estende por $93,8 \mathrm{~km}^{2}$ e contava com a estimativa de aproximadamente 16.343 habitantes no último censo do ano de 2010 e 17.153 habitantes para o ano de 2018, onde desses, 11.437 residem na área urbana e 3.713 na área rural. Ainda, de acordo com o Programa de Desenvolvimento das Nações Unidas (PDNU), Maruim apresentou IDH-M de 0,618 em 2010, situando-se na faixa de desenvolvimento humano médio (IDH-M) entre 0,600 a 0,699 (ATLAS BRASIL, 2013 A), Sergipe tem a $3^{\mathrm{a}}$ menor taxa de pobreza do Nordeste, superada apenas por Pernambuco $(40,1 \%)$ e Rio Grande do Norte (36,2\%). (IBGE, 2010 A).

Para o estudo, após assinatura dos termos de consentimento, foram distribuídos kits e coletadas 3 amostras diárias entre os dias 17 a 20 de setembro de 2018. O primeiro coletor foi entregue aos moradores 1 dia antes do início das coletas. No primeiro dia, foi recolhido 1 frasco coletor com fezes, na medida que os moradores entregavam os coletores com as amostras, recebiam novos coletores vazios para as coletas dos dias seguintes, computando um total de 3 coletores entregues e recebidos. Em seguida, foram transportados até o laboratório de Entomologia e Parasitologia Tropical da Universidade Federal de Sergipe para realizar análise macroscópica, aplicando o método de Kato-Katz, Hoffman, Pons e Janer, e o método de Ritchie (Gonçalves et al., 2016; Colombo et al., 2018). 
Os resultados obtidos, foram apresentados de forma descritiva, a concordância de positividade/negatividade por amostra entre as técnicas foi analisada pelo teste de McNemar com intervalo de confiança de 5\% e a concordância geral por meio do índice de concordância (Kappa) (Azevedo et al., 2017).

Além disso, após identificar a positividade da esquistossomose, os resultados foram associados às condições socioambientais locais visando relacioná-las à ocorrência no povoado. Todos os moradores que disponibilizaram as amostras de fezes foram convidados a responder um questionário investigativo com algumas variáveis selecionadas.

Nesse trabalho foi considerada como variável dependente os indivíduos infectados e os não infectados pelo Schistosoma mansoni e como variáveis independentes foram utilizadas variáveis sociodemográficas, socioeconômicas, condições sanitárias, contato e motivos do contato com águas naturais, educacionais e laboratoriais.

O presente estudo, foi aprovado pelo Comitê de Ética em Pesquisa da Universidade Federal de Sergipe (UFS) sob número de parecer 3.214.538.

\section{Resultados}

De acordo com os resultados achados, pode-se observar que na Tabela 1, do total de 160 indivíduos participantes do estudo 53,75\% (86) foram indivíduos do sexo feminino. Ainda do total de participantes, cerca de 51,35\% (76) vivem em zona urbana; 76,10\% (121) autodeclararam não ter renda; apenas 6,88\% (11) possuem rede de esgoto; 82,50\% (132) destinam seus lixos através de coleta pública em caminhões de lixos, no entanto, ainda 17,5\% (28) queimam, jogam no rio ou deixa seu lixo a seu aberto; 72,50\% (116) indivíduos possuem banheiro dentro de casa com água; 84,38\% (135) consomem água cujo a origem são poços, apenas $13,12 \%$ (21) possuem água encanada e a maioria, 26,88\% (43\%) dos entrevistados autodeclararam analfabetos. 
Research, Society and Development, v. 11, n. 2, e11611225523, 2022

(CC BY 4.0) | ISSN 2525-3409 | DOI: http://dx.doi.org/10.33448/rsd-v11i2.25523

Tabela 1. Caracterização socioeconômica da população amostral.

\begin{tabular}{|c|c|c|}
\hline Variável/Categoria & Frequência & Percentual (\%) \\
\hline \multicolumn{3}{|l|}{ Sexo } \\
\hline $\mathrm{F}$ & 86 & 53,75 \\
\hline M & 74 & 46,25 \\
\hline \multicolumn{3}{|l|}{ Zona } \\
\hline Rural & 72 & 48,65 \\
\hline Urbana & 76 & 51,35 \\
\hline \multicolumn{3}{|l|}{ Renda em salários mínimos } \\
\hline Sem renda & 121 & 76,10 \\
\hline Até 02 s.m. & 23 & 14,47 \\
\hline De 01 a 02 s.m. & 12 & 7,55 \\
\hline De 02 a 03 s.m. & 3 & 1,89 \\
\hline \multicolumn{3}{|l|}{ Destino do esgoto } \\
\hline Jogado na rua & 59 & 36,88 \\
\hline Canal & 2 & 1,25 \\
\hline Fossa vedada & 85 & 53,12 \\
\hline Fossa não vedada & 3 & 1,88 \\
\hline Rede de esgoto & 11 & 6,88 \\
\hline \multicolumn{3}{|l|}{ Destino do lixo } \\
\hline Céu aberto & 5 & 3,12 \\
\hline Joga no rio & 6 & 3,75 \\
\hline Queima & 17 & 10,62 \\
\hline Caminhão do lixo & 132 & 82,50 \\
\hline \multicolumn{3}{|l|}{ Tipo de banheiro } \\
\hline Não possui & 3 & 1,88 \\
\hline Comunitário sem água & 2 & 1,25 \\
\hline Comunitário com água & 3 & 1,88 \\
\hline Dentro da casa sem água & 34 & 21,25 \\
\hline Dentro da casa com água e comunitário sem água & 2 & 1,25 \\
\hline Dentro da casa com água & 116 & 72,50 \\
\hline \multicolumn{3}{|l|}{ Escolaridade } \\
\hline Analfabeto & 43 & 26,88 \\
\hline $1^{\mathrm{a}}$ a $4^{\mathrm{a}}$ série & 76 & 47,50 \\
\hline $5^{\mathrm{a}}$ a $8^{\mathrm{a}}$ série & 2 & 1,25 \\
\hline Ensino médio & 38 & 23,75 \\
\hline Ensino superior & 1 & 0,62 \\
\hline \multicolumn{3}{|l|}{ Origem da água de consumo } \\
\hline Cisterna & 4 & 2,50 \\
\hline Poço & 135 & 84,38 \\
\hline Água encanada & 21 & 13,12 \\
\hline
\end{tabular}

Fonte: Autoria própria.

Já na Tabela 2, é possível fazer um comparativo dos resultados e parasitos achados através de 3 análises distintas, o Kato-Katz, o Ritchie, o Hoffman, Pons e Janer. Foi encontrado uma maior prevalência de casos positivos para Schistosoma 
mansoni (48,12\%), ancilostomídeos $(4,38 \%)$ e Hymenolepis nana $(2,50 \%)$ quando analisado sob a técnica de Kato -Katz, os helmintos Enterobius vermicularis (5,62\%), Trichuris trichiura $(36,25 \%)$ e Strongyloides stercoralis $(5,00 \%)$ mostrou-se mais encontrado quando utilizado a técnica de Ritchie.

Tabela 2. Resultados dos parasitos e comparativo entre os métodos.

\begin{tabular}{|c|c|c|c|}
\hline Parasitos & $\begin{array}{c}\text { Kato-Katz } \\
\text { FA/FR (\%) }\end{array}$ & $\begin{array}{c}\text { Ritchie } \\
\text { FA/FR (\%) }\end{array}$ & $\begin{array}{c}\text { Hoffman, Pons e Janer } \\
\text { FA/FR (\%) }\end{array}$ \\
\hline \multicolumn{4}{|c|}{ Schistosoma mansoni } \\
\hline Positivo & $77 / 48,12$ & $70 / 43,75$ & $74 / 46,25$ \\
\hline Negativo & $83 / 51,88$ & $90 / 56,25$ & $86 / 53,75$ \\
\hline \multicolumn{4}{|c|}{ Ancilostomideos } \\
\hline Positivo & $7 / 4,38$ & $2 / 1,25$ & $1 / 0,62$ \\
\hline Negativo & $153 / 35,62$ & $158 / 98,75$ & $159 / 99,38$ \\
\hline \multicolumn{4}{|c|}{ Trichuris trichiura } \\
\hline Positivo & $20 / 12,50$ & $58 / 36,25$ & $46 / 28,75$ \\
\hline Negativo & $140 / 87,50$ & $102 / 63,75$ & $114 / 71,25$ \\
\hline \multicolumn{4}{|c|}{ Enterobius vermicularis } \\
\hline Positivo & $1 / 0,62$ & $9 / 5,62$ & $4 / 2,50$ \\
\hline Negativo & $159 / 99,38$ & $151 / 94,38$ & $156 / 97,50$ \\
\hline \multicolumn{4}{|c|}{ Strongyloides stercoralis } \\
\hline Positivo & $0 / 0,00$ & $8 / 5,00$ & $6 / 3,33$ \\
\hline Negativo & $160 / 100,00$ & $152 / 95,00$ & $154 / 96,67$ \\
\hline \multicolumn{4}{|c|}{ Hymenolepis nana } \\
\hline Positivo & $4 / 2,50$ & $3 / 1,94$ & $4 / 2,50$ \\
\hline Negativo & $156 / 97,50$ & $152 / 98,06$ & $156 / 97,50$ \\
\hline
\end{tabular}

Fonte: Autoria própria.

\section{Discussão}

As helmintíases estão entre as principais causas de problemas fisiológicos e sociais em todo o mundo. A prevalência destes parasitos é alta em comunidades pobres de regiões tropicais, com condições socioeconômicas e higiênico-sanitárias precárias (Santos, et al 2020). A investigação de infecções parasitárias intestinais é pré-requisito para o desenvolvimento de estratégias de controle adequadas, já que é um importante indicador das condições de saúde e saneamento básico em que vive uma população (Rebolla, 2012). O diagnóstico parasitológico das infecções causadas por estes helmintos é significativo para o tratamento do paciente e controle da disseminação destes agentes (Silva, et al., 2015).

A aplicação de diferentes métodos de exames de fezes torna-se necessária tendo em vista a variabilidade morfológica e biológica apresentada pelos parasitos. Nem sempre uma técnica apropriada ao encontro de ovos será eficiente na detecção de larvas de helmintos (Mendes, 2005). As três técnicas que foram empregadas neste estudo permitiram a detecção de diversos ovos e larvas de parasitos. 
Para a pesquisa de elementos parasitários nas fezes, a técnica de Hoffman, Pons e Janer (HPJ), é atualmente o método de sedimentação por força gravitacional utilizado com maior frequência nas rotinas laboratoriais no Brasil, e mais eficiente na detecção de ovos pesados como: ovos de cestódeos (Taenia spp.) e trematódeos (Schistosoma spp.), e alguns laboratórios a utilizam também para a observação de ovos leves como: Trichuris trichiura, Ancylostoma duodenale (Santos et al., 2020). Santos e colaboradores (2020) ainda enfatizam que o HPJ é um método de execução simples, não altera a viabilidade dos ovos, porém é uma técnica que necessita de um tempo maior para a sua execução e leitura e acumula muitos detritos dificultando a observação e identificação dos parasitos.

Com relação a sensibilidade dos métodos laboratoriais utilizados na pesquisa, foi evidenciado valores distintos com relação a identificação do tipo de agente patogênico para cada método.

Utilizando a técnica de HPJ, os achados deste estudo corroboram com os encontrados por Katagiri e Oliveira-Sequeira (2010), onde permitiu identificar ovos até mesmo do parasito Enterobius vermicularis. Este método não é específico para identificação do ovo deste parasito, segundo a literatura o método Graham (o da fita gomada ou fita adesiva) é o mais sensível para obtenção de ovos de parasitos do gênero Enterobius (Ferreira, 2012; Zeibig, 2014).

No mesmo estudo realizado por Katagiri e Oliveira-Sequeira (2010), utilizando a mesma técnica foram encontrados ovos do cestódeo Taenia spp. Este método mostra o quão é importante, pois pode recuperar ovos de parasitos não comuns, por se tratar de um método de sedimentação e geralmente para o encontro deste parasito a técnica de HPJ convencional consegue recuperá-la.

Em nossas análises utilizando o método convencional HPJ, identificamos 3,33\% de pacientes parasitados com Strongyloides stercoralis esses achados vão de encontro com os encontrados por Seixas et al, (2012) que relataram também o encontro de Strongyloides stercoralis em 2,5\% das amostras e Ancylostoma duodenale em 1,5\%.

Em relação a técnica de Kato-Katz (KK), os resultados obtidos nesse estudo são diferentes dos descritos por Azevedo et al., 2017 onde a técnica apresentou menor capacidade de detecção entre as técnicas analisadas. Tal fato pode ter sido devido à leitura de uma única lâmina nessa técnica. Já Tarafder et al., (2010) em seu estudo aponta a técnica de Kato-Katz como melhor opção para o diagnóstico de geohelmintos, como Trichuris trichiura, o que está em acordo com os resultados desse estudo.

Dentre as diversas técnicas utilizadas para a análise parasitológica, destaca-se o método de Ritchie, que tem base em uma metodologia de reconhecida eficiência para o diagnóstico de helmintos nas fezes, pela realização de uma centrífugo-sedimentação com o uso do éter etílico (C4H10O) e formaldeído (HCHO) (Almeida et al., 2009).

Nos resultados explanados por Azevedo (2016) a técnica de Ritchie apresentou uma maior eficiência no diagnóstico de helmintos (20,1\%). Carvalho et al., (2002) observaram que a técnica de Ritchie modificada por Young (1979) apresentou melhor resultado como técnica única. Nesse estudo Ritchie também obteve ótimos resultados para detecção de helmintos indo de encontro com os resultados descritos acima.

Nossos resultados vão de encontro aos achados por Santos et al., (2021) em Maruim, no estado de Sergipe de acordo com o exposto, estes, mostraram que a técnica de Ritchie possuía uma adequada sensibilidade a presença de ovos de helmintos nas fezes.

Santos et al., (2021) apresentam resultados similares aos expostos neste estudo, segundo estes, em relação as condições sociais e econômicas, maioria das pessoas se autodeclaram não haver salários fixos constatando uma alta probabilidade de serem autônomos e/ou viverem da pesca. Sena et al., (2020) em seu estudo, mostram elevadas taxas de prevalência intestinal em comunidades ribeirinhas esses resultados corroboram com os resultados obtidos no presente estudo e denotam uma precariedade das condições de saneamento básico às quais essas comunidades estão submetidas, que associada ao baixo poder econômico e baixa escolaridade, contribuem para o cenário de caos instalado na saúde e na dignidade dessas populações.

Apesar de 132 pessoas serem contempladas com coleta de lixo, ainda 28 destinam seus lixos de forma totalmente inadequada (queima, joga no rio ou deixa a céu aberto), 85 ainda possuem fossas, mesmo que vedada, apenas 11 tem rede de 
esgoto considerada adequada o que é muito pouco, e isso inclusive pode acarretar danos a lençóis freáticos contaminando-os e assim também a população, já que 135 ainda fazem utilização de poços devido à falta de água encanada.

Os achados acima mencionados evidenciam e vão de encontro com os resultados achados por Meira et al., (2021) nas regiões nordeste, sudeste e centro-oeste do Brasil. No estado da Bahia, observou-se que as amostras foram positivas para presença de parasitos em 70,7\% dos casos mostrando maior prevalência entre helmintos e protozoários no geral. Contudo, em relação aos helmintos, notou-se maior prevalência de: Ascaris lumbricoides (48,3\%), Trichuris trichiura (10,3\%) e Ancilostoma duodenale $(6,9 \%)$.

Sena et al., (2020); Meira et al., (2021); Maia et al., (2016), Lodo et al., (2010) e Santos et al., (2021) afirmam que entre as principais causas de altas prevalências por helmintos, destacam-se condições habitacionais e sanitárias precárias, ingestão de água e alimentos contaminados, baixas condições socioeconômicas e um baixo nível de escolaridade. É importante salientar que as helmintíases fazem parte das doenças tropicais negligenciadas, sendo assim, não podem ser vistas apenas no âmbito biológico, diversos aspectos podem condicionar o aparecimento das mesmas em populações mais vulneráveis. Sob esse ponto de vista, é necessária a intersecção entre o processo saúde doença e as iniquidades em saúde (Siqueira et al., 2021)

\section{Conclusão}

De acordo com os dados obtidos neste estudo, evidencia-se que os métodos aqui apresentados mostraram resultados semelhantes entre as técnicas utilizadas, entretanto, com base nesses mesmos dados verificou-se, que somente a utilização de um método diagnóstico não é eficiente para a identificação das mais variadas espécies de helmintos.

As técnicas se mostram bastante eficiente na identificação dos parasitas, porém nem todos os parasitos foram identificados simultaneamente pelas técnicas. Com base nesta informação, conclui-se que uma técnica pode complementar a outra no diagnóstico parasitário aumentando assim sua sensibilidade.

Afirmando o que foi dito logo acima, enfatiza-se que em laboratórios de rotina seria importante realizar mais de um método de diagnóstico para detectar os helmintos, principalmente quando há baixa carga parasitária. Os métodos utilizados se mostraram um meio rápido, sendo exames não invasivos e úteis para levantamentos de perfil diagnóstico e epidemiológico.

\section{Referências}

Almeida, A. F et al., (2009). Adaptation of Ritchie Method for diagnosing Helminthes and Protozoa in samples of sewage silt with chemical substances minimization. O Mundo da Saúde, São Paulo.

Amrante, A. F. T.; Ragozo, A. M. A.; Silva, B. F. 9 (2014). Helmintos. Editora Unesp, São Paulo. Pag. 6-7.

Azevedo, E. P. (2016). Diagnóstico coproparasitológico em laboratório de análises clínicas: comparação de técnicas e custo de implantação. (Trabalho de Conclusão de Curso) - Programa de Graduação de Biomedicina, da Universidade Federal Fluminense.

Azevedo, E. P. et al., (2017). Parasitological diagnosis in fecal samples in the laboratory of clinical analyses: comparison of techniques and cost of implantation. RBAC.

Carvalho, O. S. (2002). Prevalência de helmintos intestinas em 3 mesorregiões de minas gerais. Revista da Sociedade Brasileira de Medicina Tropical 35, (6) p. 597-600, nov-dez.

Ferreira, M. U. Parasitologia Contemporânea. 1. ed. Rio de Janeiro: Guanabara Koogan, 2012.

Hernandes, J. C et al., (2018). The comparison between two parasitological techniques for the detection of enteroparasites in waste pickers from the south of Brazil. Semina, v. 39, n. 1, p. 29-40.

Katagiri S, \& Oliveira-Sequeira, T C G. (2010). Comparison of three concentration methods for the recovery of canine intestinal parasites from stool samples. Experimental Parasitology. San Diego: Academic Press Inc. Elsevier B.V.

Katz, N. (2018) Inquérito Nacional de Prevalência da Esquistossomose mansoni e Geo-helmintoses/Naftale Katz. - Belo Horizonte: CPqRR, 22. ed. - 614.5.

Lodo, M. et al., (2010) Prevalência de enteroparasitos em município do Interior Paulista. Rev Bras Crescimento Desenvolvimento Hum. 20, (3) p. 769-777 
Research, Society and Development, v. 11, n. 2, e11611225523, 2022

Maia, C. V. A.; \& Hassum, I. C. (2016). Parasitoses intestinais e aspectos socio sanitários no Nordeste Brasileiro no século XXI: Uma Revisão De Literatura. Revista Brasileira de Geografia Médica e da Saúde, Hygeia 12, (23), p. 20-30.

Meire de, R. Z C., Takizawa, M. G. M. H., Zgoda, I.; \& Reolon, L. T. (2021). Comparação entre a prevalência de parasitoses intestinais no brasil: revisão sistemática. Revista Thêma et Scientia - Vol. 11, no 1E.

Mendes. R. C. et al. (2005). Estudo comparativo entre os métodos de Kato-Kazt e coprotest. Revista da Sociedade Brasileira de Medicina Tropical. Uberaba, vol. 38 n. 2, p. $178-180$

Moreira, M. L., Valadão, A. F., \& Martins, J. (2011). Prevalence of mansonic schistosomiasis and associated factors to its occurrence in students from the rural area of Inhapim-MG, 2008. Rev. Bras. Farm.(4):53-8.

Motta, J. L.; Franco, S. F.; \& Pavanelli, M. F. (2020). Comparação do perfil epidemiológico entre indivíduos parasitados de Campo Mourão e Peabiru, Paraná. Saúde em Redes.

Rebolla, M. F. (2012). Inquérito parasitológico, comparação de técnicas de diagnóstico fecal, controle e prevenção de Giardia em creches e pré-escolas, São Sebastião da Grama, São Paulo. (Dissertação de mestrado) - Programa de pós graduação em parasitologia da Universidade Estadual de Campinas.

Santos, A. H.C., Barbosa, L., Siqueira, T. S., Souza, M. R., Celestino, A. O., Santos, A. F.; \& Araújo, K. C. G. M. (2021). Prevalence and risk factors associated with the infection of Schistosomiasis mansoni and enteroparasitosis in an endemic area, Sergipe, Brazil. Research, Society and Development, v. 10, n. 5 , e26310514538.

Santos, K. R., Ciro, E. R., Miranda, L. S. R., Lino, M. N., \& Júnior, S. C. S.(2020). Comparação entre três técnicas coproparasitológicas na investigação de parasitos intestinais de seres humanos. REAS/EJCH.

Seixas M T L, et al. (2012) Avaliação da frequência de parasitos intestinais e do estado nutricional em escolares de uma área periurbana de Salvador, Bahia, Brasil. Revista de Patologia Tropical. 40(4): 304-14.

Sena, L. W. P et al., (2020) Prevalence of enteroparasitosis in a riverside community in the state of Pará, Brazil. REAS/EJCH,

Silva, A.O. et al., (2015). Epidemiologia e prevenção de parasitoses intestinais em crianças das creches municipais de Itapuranga-GO. Revista Faculdade Montes Belos.; 8 (2): 1-17.

Silva, C. P., Aranda, K. R. S., Barnabe, A. S., Mello, T. R. C., \& Ferraz, R. R. N. (2020). Performance profile of coproscopic techniques Coproplus ${ }^{\circledR}$ and Hoffman, Pons and Janner in the diagnosis of giardiasis. Rev. Epidemiol. Controle Infecç. Santa Cruz do Sul.

Táparo, C. V et al., (2006). Comparação entre técnicas coproparasitológicas no diagnóstico de ovos de helmintos e oocistos de protozoários em cães. Revista Brasileira de Parasitologia Veterinária, vol. 15, núm. 1.

Tarafder, M. R, et al., (2010). Estimating the sensitivity and specificity of KatoKatz stool examination technique for detection of hookworms, Ascaris lumbricoides and Trichuris trichiura infections in humans in the absence of a 'gold standard'. Int. j. parasitol.;40(4): 399-404.

Tibiriça, S. H. C et al., (2009). Validação do número de lâminas para realização do método de sedimentação espontânea das fezes. HU Revista, Juiz de Fora, v. 35, n. 2, p. 105-110.

Young, K. H., Bullock, S. L., Melvin, D. M., \& Sprulll, C. L. (1979) Ethyl acetate as a substitute for diethyl ether the formalin-ether sedimentation technique. $J$ Clin Microbiol, v. 10, n. 6, p. 852-853.

Siqueira, T. S., Celestino, A. O., Santos, A. H. C., Souza, M. do R., Santos, A. F., Barbosa, L., \& Silva, J. R. S. (2021). Epidemiological indicators of leprosy in Sergipe: A look towards the Social Determinants of Health. Research, Society and Development, [S. l.], v. 10, n. 4, p. e38610414367. DOI: 10.33448/rsdv10i4.14367. Disponível em: https://rsdjournal.org/index.php/rsarticle/view/14367. Acesso em: 4 jan. 2022. 